\title{
REVIEWS
}

Check for updates

\section{Cytokine release syndrome and associated neurotoxicity in cancer immunotherapy}

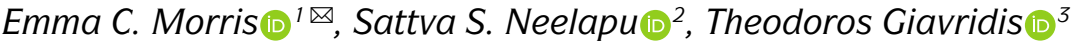 \\ and Michel Sadelain ${ }^{3}$
}

Abstract |A paradigm shift has recently occurred in the field of cancer therapeutics. Traditional anticancer agents, such as chemotherapy, radiotherapy and small-molecule drugs targeting specific signalling pathways, have been joined by cellular immunotherapies based on T cell engineering. The rapid adoption of novel, patient-specific cellular therapies builds on scientific developments in tumour immunology, genetic engineering and cell manufacturing, best illustrated by the curative potential of chimeric antigen receptor (CAR) T cell therapy targeting CD19-expressing malignancies. However, the clinical benefit observed in many patients may come at a cost. In up to one-third of patients, significant toxicities occur that are directly associated with the induction of powerful immune effector responses. The most frequently observed immune-mediated toxicities are cytokine release syndrome and immune effector cell-associated neurotoxicity syndrome. This Review discusses our current understanding of their pathophysiology and clinical features, as well as the development of novel therapeutics for their prevention and/or management.

'Department of Immunology, Institute of Immunity and Transplantation, University College London, London, UK.

${ }^{2}$ Department of Lymphoma and Myeloma, The University of Texas MD Anderson Cancer Center, Houston, TX, USA.

${ }^{3}$ Center for Cell Engineering, Sloan Kettering Institute, Memorial Sloan Kettering Cancer Center, New York, $N Y$, USA.

凶e-mail:e.morris@ucl.ac.uk https://doi.org/10.1038 s41577-021-00547-6
Adoptively transferred, tumour antigen-specific T cells genetically engineered to express chimeric antigen receptors (CARs) ${ }^{1}$ have achieved durable clinical responses in patients with some types of cancer, particularly CD19-expressing refractory and relapsed $B$ cell malignancies ${ }^{2-6}$. CARs are synthetic antigen recognition receptors, which include an antibody-derived single-chain variable fragment $(\mathrm{scFv})$, hinge and transmembrane domains and intracellular signalling domains. The intracellular signalling domains - typically, the $\mathrm{CD} 3 \zeta$ signalling domain plus co-stimulatory signalling domains such as those from CD28 (REF.7) and 4-1BB (also known as TNFRSF9) ${ }^{8}$ - together with the cellular phenotype of the engineered $\mathrm{T}$ cell influence the specific cytokine secretion profile and in vivo proliferative capacity of the CAR T cells once activated by antigen-expressing target cells.

Despite their clinical success, the use of CAR T cells can result in significant toxicities that are directly associated with the induction of powerful immune effector responses. Two major toxicities arising from CAR $T$ cells were not revealed in early mouse models but were eventually observed in clinical trials - cytokine release syndrome (CRS) and immune effector cell-associated neurotoxicity syndrome (ICANS; often referred to as neurotoxicity). CRS usually starts with fever and constitutional symptoms such as rigours, malaise and anorexia ${ }^{9}$. The fever can be of high grade and persist for several days. In severe cases, CRS manifests with other features of a systemic inflammatory response, including hypotension, hypoxia and/or organ dysfunction. Organ dysfunction may occur secondary to the hypotension or hypoxia but can also result from the direct effects of cytokine release. Dysfunction of all major organ systems, including cardiac, pulmonary, hepatic, renal and gastrointestinal systems, has been observed in patients with CRS. However, this organ dysfunction is preventable or reversible in most patients if the symptoms and signs of CRS are promptly recognized and managed ${ }^{9-15}$.

ICANS typically manifests as a toxic encephalopathy and starts with word-finding difficulty, confusion, dysphasia, aphasia, impaired fine motor skills and somnolence ${ }^{14,16,17}$. In more severe cases, seizures, motor weakness, cerebral oedema and coma have been noted. The majority of patients who develop clinical features of ICANS will have had preceding CRS. CRS can therefore be considered an 'initiating event' or cofactor for ICANS. ICANS generally occurs after the symptoms of CRS have subsided (FIG. 1), although, less frequently, concurrent presentation of CRS and ICANS can occur ${ }^{1,18}$. Similar to CRS, ICANS is reversible in most patients with no permanent neurological deficits ${ }^{10,11,14-17}$. 


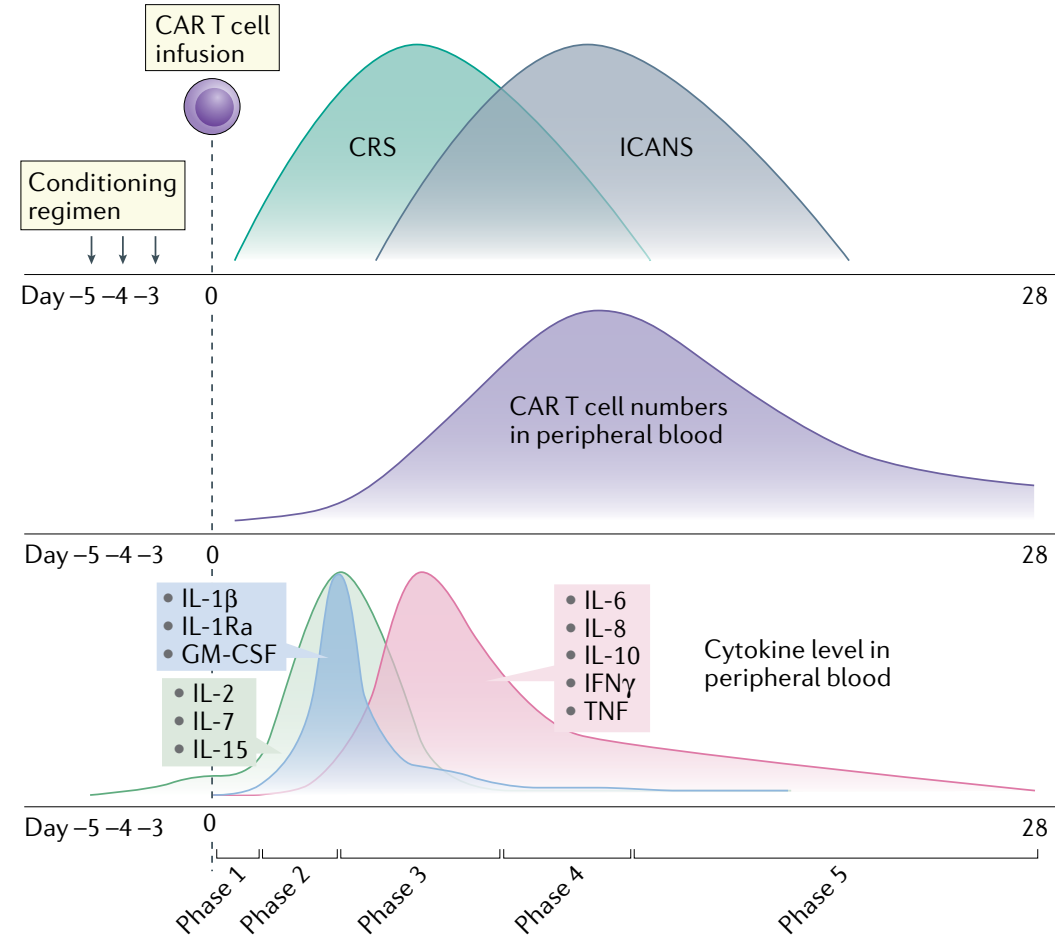

Fig. 1 | Schematic diagram showing a relative timescale for the onset and duration of CRS and ICANS. Also shown are the kinetics of chimeric antigen receptor (CAR) T cell proliferation and cytokine levels in peripheral blood. Conditioning chemotherapy, such as with cyclophosphamide and fludarabine, was given for a period of 3-5 days before infusion of CD19-directed CAR T cells (CD19CAR T cells; day 0) in patients with lymphoma or leukaemia. The onset and peak of cytokine release syndrome (CRS) generally precede those of immune effector cell-associated neurotoxicity syndrome (ICANS), with CRS generally occurring in the first week after CAR T cell infusion and ICANS occurring in the second week after infusion. CAR T cell numbers peak in the peripheral blood 1-2 weeks after infusion. Following activation-induced cell death of effector CAR T cells, some of the CAR T cells may persist as long-term memory T cells. Levels of homeostatic cytokines, such as IL-2, IL-7 and IL-15, may increase after conditioning therapy owing to lymphodepletion and the elimination of cytokine sinks, with a further increase in cytokine levels observed after CAR T cell infusion. These cytokines promote the survival and proliferation of CAR T cells. Levels of IL-1 $\beta$ and its natural antagonist IL-1 receptor agonist (IL-1Ra), as well as of granulocyte-macrophage colony-stimulating factor (GM-CSF), tend to peak earlier than other pro-inflammatory cytokines, which suggests that these cytokines might have a role in initiating the inflammatory cascade, together with IL-6. The pathophysiology of CRS can be divided into five main phases as indicated (see main text for details). IFN $\gamma$, interferon- $\gamma$; TNF, tumour necrosis factor.

Poor-risk malignant disease Subtypes of malignancy (cancer) that have a poor prognosis with conventional therapies.

Blood-brain barrier (BBB). A network of blood vessels and tissues that form a functional barrier to prevent harmful substances from entering the brain

Activation-induced cell death In peripheral T cells, death that is often the result of engagement of cell death pathways (for example, CD95CD95L) that are upregulated during immune activation.
Currently, most clinical applications of CAR T cells are for patients with poor-risk malignant disease, and within the field of haemato-oncology, most patients have been treated with CD19-directed CAR T cells (CD19CAR T cells), which are therefore the focus of this Review. However, it should be noted that similar toxicities to those described here have been observed in multiple clinical indications for a wide range of CAR $\mathrm{T}$ cell specificities. Rapid progress is underway in the development of genetically modified immune cells for non-malignant indications such as autoimmune diseases and solid organ transplant tolerance. In these cases, the risk-benefit balance is less favourable for potentially toxic therapies, requiring improved management of toxicities or lower toxicity profiles of the immunotherapeutic interventions. As a result, it is important to better understand the clinical features and pathophysiological mechanisms of CRS and ICANS. Here, we discuss what is known so far and address how this might impact the development of novel therapeutics for the prevention and/or management of these toxicities. A greater understanding of CRS and ICANS also has wider relevance to other systemic, cytokine-mediated inflammatory diseases, such as septic shock, macrophage activation syndrome (BOX 1), neuroinflammatory disease and infection with emerging pathogens such as SARS-CoV-2 (BOX 2), as well as to other therapeutic interventions such as the potential role of IL-1 blockade in cancer immunotherapy (BOX 3). As detailed below, our understanding of the pathophysiology of CRS and ICANS associated with CAR T cell therapy started with bedside to bench research but now has been further extended by detailed investigation in animal models.

\section{Pathophysiology of CRS}

The pathophysiology of CRS can be divided into five main phases. Phase 1 involves the trafficking of CAR T cells to the tumour site following their infusion into a patient and CAR-mediated recognition of antigen-expressing target cells. In phase 2, there is proliferation of CAR T cells at the tumour site, in situ cytokine production by both activated CAR T cells and cellular components of the tumour microenvironment, activation of 'bystander' endogenous immune cells, direct and indirect tumour cell killing, and onset of CRS. In phase 3 , there is an increase in cytokine levels and expansion of CAR T cell populations in the peripheral blood, which is associated with a systemic inflammatory response. This leads to endothelial injury and vascular leakage in multiple tissues and organs, and their associated effects including hypoxia, hypotension and/or organ damage. The diffusion of cytokines and transmigration of CAR $\mathrm{T}$ cells, endogenous $\mathrm{T}$ cells and peripherally activated monocytes into the cerebrospinal fluid (CSF) and central nervous system (CNS) in phase 4, including breakdown of the blood-brain barrier (BBB), coincides with the onset of ICANS. In phase 5, activation-induced cell death of $\mathrm{T}$ cells following eradication of the tumour results in decreased serum cytokine levels and a reduced systemic inflammatory response, the end of CRS and/or ICANS symptoms and, potentially, the persistence of long-term memory CAR T cells. The relative timings for the onset and duration of CRS and ICANS are shown in FIG. 1.

CRS had not been anticipated from preclinical studies of CD19CAR T cells ${ }^{8,19,20}$ and was only recognized after phase I clinical trials of CAR T cells were initiated ${ }^{4,7}$. Although the resulting fever and hypotension in patients were immediately associated with increased serum cytokine levels, the mechanisms underlying this occasional outcome, which is more common in patients with high tumour burden ${ }^{4,7,21}$, were initially unclear. It is now understood that nearly all patients treated with CD19CAR T cells develop some level of CRS; in the registration trials, up to one-third of patients with B cell acute lymphoblastic leukaemia developed severe CRS and up to one-half of patients developed ICANS ${ }^{22}$, although the real-world rates of CRS and ICANS may be lower than this. The empirical testing of different 
$\overline{\text { CD19+ lymphoma xenograft }}$ model

A lymphoma model in which immunodeficient mice are engrafted with human lymphoma cells positive for the CD19 surface antigen

Humanized NSG mice An immunodeficient mouse strain (NSG) reconstituted with human immune cells; in mice are engineered with transgenes encoding human haematopoietic cytokines. some iterations of this model

blocking antibodies soon identified IL-6 as a crucial mediator of $\mathrm{CRS}^{4,7}$ and tocilizumab, a monoclonal antibody that blocks signalling through the IL- 6 receptor (IL-6R), became a staple of CRS management ${ }^{3,23}$. As activated T cells can produce IL-6, it was initially assumed that the CAR T cells themselves were its main source, even though some observations suggested additional possible contributors ${ }^{3,24}$. However, subsequent investigations, discussed below, have identified macrophage and monocyte lineage cells as the source of IL-6. It is noteworthy that, despite there seeming to be significant differences in some studies between the activation kinetics and cytokine-producing abilities of CAR T cells including either CD28 or 4-1BB signalling domains s $^{4,7,10,21,25,26}$, the CRS associated with either type of CAR is highly similar $^{12,27,28}$. Although the CRS induced by CD28-based CAR T cells tends to develop earlier than that initiated by $4-1 \mathrm{BB}-$ based CAR T cells, the cytokine profiles observed in patient serum hardly differ with regards to peak levels of cytokines and chemokines, which is consistent with a common pathophysiological mechanism. Levels of IL-6, IL-8, IL-1 receptor antagonist (IL-1Ra), CC-chemokine ligand 2 (CCL2) and CCL3, which are not primarily $\mathrm{T}$ cell-derived products, are commonly increased ${ }^{7,17,25,29}$, which indicates that there is likely to be a common mechanism of CRS that extends beyond the CAR T cells themselves and involves host cells.

Box 1 | Similarities between cytokine release syndrome, haemophagocytic lymphohistiocytosis and macrophage activation syndrome

Haemophagocytic lymphohistiocytosis $(\mathrm{HLH})$ and macrophage activation syndrome (MAS) are clinical syndromes of pathological hyperinflammation and uncontrolled macrophage activation that are usually associated with triggers such as viral infections, rheumatological diseases or inherited defects in T cell and natural killer cell functions ${ }^{117-119}$. A connection between cytokine release syndrome (CRS) and MAS has been clear since the first recognition of CRS. Patients with either MAS or chimeric antigen receptor (CAR) T cell-mediated CRS were both noted to have biochemical profiles consistent with hyperinflammation, including hyperferritinaemia, prolonged cytopenia, fever, coagulopathy and liver function abnormalities ${ }^{31,120}$. Since then, prolonged life-threatening systemic inflammatory responses resembling MAS and $\mathrm{HLH}^{14}$ have been observed following CD19-directed CAR T cell (CD19CAR T cell) infusion or use of CD19-directed blinatumomab, a bispecific T cell engager therapy ${ }^{121}$, and after CD22CAR T cell therapy ${ }^{31}$

More recently, a syndrome of late HLH or MAS has been described that does not clearly overlap with CRS. A phase I clinical trial testing CD22CAR T cells in patients with relapsed or refractory $B$ cell acute lymphoblastic leukaemia after prior treatment with CD19CAR $T$ cells observed an increased rate of inflammatory toxicities ${ }^{30,31}$. These HLH/MAS-like toxicities only occurred in patients who had experienced CRS and the onset of $\mathrm{HLH}$-like features occurred a median of 14 days after CD22CAR T cell infusion, typically after the resolution of CRS. The second phase of this biphasic inflammatory response was associated with re-expansion of CAR T cell populations. This temporal dissociation between CRS and late HLH/MAS is suggestive of a unique pathophysiology, and further studies into IL-1 receptor (IL-1R1) blockade are warranted in this context. T cell factors may be important. Significantly higher serum concentrations of IL-6, interferon- $\gamma$ (IFN $\gamma$ ), IL-8, IL-15, IL-10, tumour necrosis factor and IL-1 $\beta$ were documented following the infusion of $\mathrm{CD} 22 \mathrm{CAR}$ T cell products that included a $\mathrm{CD} 8^{+}$and $\mathrm{CD} 4^{+}$selection step in the manufacturing process, which was also associated with greater HLH/MAS-like toxicity.

A preclinical mouse model of late-onset CAR T cell-mediated toxicities has recently been published ${ }^{122}$ using wild-type or perforin-deficient CAR T cells. Recipients of perforin-deficient CAR T cells developed an HLH-like hyperinflammatory response associated with re-expansion of the CAR T cell population in the absence of detectable target antigen. Interestingly, the perforin-deficient CAR T cells upregulated a complex pro-inflammatory cascade, including increased circulating IFN $\gamma$ levels, upregulation of IL-1 family members and secondary inflammatory changes reminiscent of gene expression signatures reported in patients with $\mathrm{HLH}$ and MAS ${ }^{123,124}$.
A schematic representation of the pathophysiology of CRS is shown in FIG. 2

The similarities between CRS and systemic inflammatory response syndromes such as haemophagocytic lymphohistiocytosis and macrophage activation syndrome are discussed in BOX 1. Although these and other later-onset inflammatory toxicities have been observed independently of CAR T cell specificity, the published data suggest that they occur more frequently with CD22CAR T cells, which were developed to target tumour relapse secondary to CD19 antigen escape ${ }^{30-32}$.

Cellular interactions and molecular mediators. Two mouse models eventually showed that CRS results from a multicellular network involving CAR T cells and host cells, with central involvement of macrophage and monocyte lineage cells. One study showed that in a CD 19+ lymphoma xenograft model, several endogenous cell populations at the tumour site, including dendritic cells, monocytes and macrophages, produced IL-6, with macrophages vastly outnumbering the other cell types $^{29}$. In this xenogeneic setting, levels of mouse IL-6 greatly exceeded levels of human IL- 6 produced by the CAR T cells. IL- 6 production was not induced at distal tumour-free sites, which supports the idea that CRS originates locally but produces a systemic pathology. The other study ${ }^{33}$ showed in humanized NSC mice using a patient-derived leukaemic cell line ${ }^{34}$ that depletion of phagocytic cells with clodronate or CAR T cell-mediated targeting prior to therapeutic CAR T cell administration abrogated IL- 6 production and overt CRS. Single-cell RNA-sequencing data of leukocytes isolated during CRS confirmed that monocyte lineage cells are the origin of IL-6. In both models and in accordance with clinical experience, blockade of IL-6 largely mitigated CRS-associated toxicity ${ }^{29,33}$. Both studies further revealed that IL-1, a monocyte- and macrophage-derived cytokine, is also a potent driver of CRS-associated toxicity.

Potential triggers of macrophage recruitment or activation during CRS are emerging. CAR T cells must themselves be activated to induce cytokine production by myeloid cells ${ }^{29}$, which is in agreement with the clinical observation that CRS is mild or absent in patients who do not respond to CAR T cell therapy $4,10,11,28,35$. It is uncertain whether contact-dependent interactions between CAR T cells and host myeloid cells are required. CD40-CD40L interactions, although not required to elicit CRS, can exacerbate macrophage activation, thereby increasing IL- 6 production and aggravating $\mathrm{CRS}^{29}$. It remains to be determined what other contact-dependent mechanisms, if any, may be crucial for CRS development or amplification. T cell surface molecules such as the integrin LFA1 and the co-stimulatory molecule CD28 interact with cognate receptors (ICAM1 and CD80 or CD86, respectively) that are robustly expressed by myeloid cells ${ }^{36,37}$. The CD28-CD80/CD86 axis may be of particular interest in the context of CRS given its reported bidirectional signalling and potential role in inducing IL- 6 production by myeloid cells ${ }^{37}$. Blockade of these interactions could potentially reduce CRS severity ${ }^{38,39}$. 


\section{Box 2 |Cytokine release syndrome and COVID-19}

Recently, cytokine release syndrome (CRS) has attracted a lot of interest in the context of COVID-19 caused by SARS-CoV- 2 infection. A significant number of patients with COVID-19 develop immune complications and respiratory debilitation known as acute respiratory distress syndrome (ARDS) that resembles CRS-related ARDS. In both cases, respiratory support through intubation may be required and the pathophysiology involves cytokine hypersecretion, dysregulated macrophage activation, lymphopenia and subsequent cardiovascular effects ${ }^{125}$. Indeed, in one meta-analysis of clinical outcomes of COVID-19, $93 \%$ of fatal cases were attributed to respiratory failure and/or cardiovascular failure ${ }^{126}$, pathologies that are also commonly observed in chimeric antigen receptor (CAR) T cell-derived CRS. Intriguingly, in this study, IL-6 levels were found to be differentially increased in those patients who eventually died from these complications. Another study also showed that cytokine hypersecretion strongly correlates with fatal outcomes in COVID-19 (REF.127); levels of cytokines and their signalling mediators, such as IL-6, soluble IL-2 receptor, IL-8, IL-10 and tumour necrosis factor, were found to be differentially increased in severe cases, stratified by increasingly impaired respiratory fitness ${ }^{128}$, a cytokine profile similar to that observed in CAR T cell-induced CRS ${ }^{13}$.

Given that the IL-6 receptor (IL-6R)-blocking antibody tocilizumab is approved for the treatment of patients with CAR T cell-derived CRS and that increased levels of IL- 6 have been observed in patients with severe COVID-19, tocilizumab has been tested at various centres for its efficacy in treating COVID-19-related cytokine hypersecretion. In some studies, use of tocilizumab reduced the length of and/or need for ventilation ${ }^{128}$, accelerated fever resolution and acute phase protein $\operatorname{control}^{129}$, produced a statistically significant reduction in risk for mortality ${ }^{128}$ or resulted in a beneficial survival trend ${ }^{130}$. A recently published larger study concluded that tocilizumab reduced the likelihood of progression to mechanical ventilation or death but did not improve survival ${ }^{131}$. Some studies have suggested that clinical benefit is restricted to patients requiring mechanical ventilation ${ }^{132}$. It should be noted that these studies were carried out rapidly in response to the evolving pandemic and, therefore, that confounding factors, such as the co-treatment of patients with other modalities such as corticosteroids, hydroxychloroquine and antiretrovirals, may complicate interpretation of the results. Moreover, these studies were not blinded and patient allocation to the tocilizumab plus standard of care arm versus the standard of care alone arm was, in some cases, disproportionate in terms of disease severity and ventilation requirement. In early 2021, the Medicines and Healthcare products Regulatory Agency (MHRA) in the United Kingdom issued guidance to encourage the use of tocilizumab and sarilumab (another IL-6R-blocking antibody), especially for the treatment of critically ill patients receiving supportive ventilation. Interestingly, serum levels of IL- $1 \beta$ were not noted to differ between patients with moderate and severe COVID-19 (REF. ${ }^{127}$ ). Nonetheless, based on prior knowledge of IL-1 biology and recent preclinical findings regarding its role in CAR T cell-induced CRS, four clinical trials recently reported a benefit from treatment of patients with COVID-19 with the IL-1 receptor antagonist anakinra. In one study that compared patients with severe COVID-19 treated with anakinra against a historical control of patients mirroring the inclusion criteria, anakinra reduced the frequency of intensive care admission for respiratory support and death in a statistically significant manner ${ }^{133}$. In another study using a retrospective patient cohort, anakinra reduced the incidence of death from COVID-19 in a statistically significant manner. Moreover, levels of the inflammatory marker C-reactive protein were reduced, as was the requirement for respiratory support concomitant with ARDS resolution ${ }^{134}$. In a third study, anakinra in combination with the corticosteroid methylprednisone led to a statistically significant reduction in mortality and risk of death in treated patients ${ }^{135}$. Lastly, in a fourth study, patients with COVID-19 who received early treatment with anakinra had a rapid resolution of fever without any deaths reported ${ }^{136}$. Additional controlled trials of both tocilizumab and anakinra will be required to confirm these results.

\footnotetext{
Acute phase response An early-onset, innate,

systemic inflammatory reaction that results from various insults such as infection and tissue injury.

SCID-beige xenograft model

Severe combined

immunodeficient (SCID)-beige

mice lack mature $B$ cells and T cells and exhibit defective natural killer cell responses, which makes them permissive to engraftment with human tumour tissue.
}

Cytokine mediators: IL-6 and IL-1. Contact-independent interactions involving the cytokines IL- 6 and IL- 1 are known to have important roles in the pathophysiology of CRS, as discussed above. IL- 6 is a pleiotropic cytokine that has both pro-inflammatory and anti-inflammatory effects. IL- 6 is primarily produced by macrophages and other cells of the myeloid lineage and can function in an autocrine manner, promoting macrophage maturation and activation in combination with other inflammatory signals. IL-6R is primarily expressed by immune cells, including microglia, and by hepatocytes ${ }^{40,41}$. IL-6 can signal and promote inflammation through both cis-signalling and trans-signalling. Signalling in trans has broad effects outside the immune system when soluble IL-6-IL-6R forms a complex with ubiquitously expressed membrane-bound gp130. For example, in addition to controlling the acute phase response, IL-6 participates in the regulation of hyperthermia, glucose metabolism, the neuroendocrine system and appetite ${ }^{41,42}$. However, the precise role of IL-6 in CRS remains elusive. IL-6 blockade can lead to the reversal of most symptoms and across-the-board cytokine downregulation in many patients ${ }^{4,7,10,11}$. In preclinical models, IL-6 was also found to mediate mortality from CRS ${ }^{29,33}$ as well as to contribute to macrophage activation through the induction of nitric oxide synthase (iNOS) and production of nitric oxide $(\mathrm{NO})^{29}$.

IL-1 is also a pleiotropic cytokine with multiple functions ${ }^{43}$. It is produced predominantly by monocytes and macrophages. The IL-1 receptor (IL-1R1) is ubiquitously expressed and responsible for transducing pro-inflammatory signalling. IL-1 can induce tissues to produce downstream pro-inflammatory cytokines, such as IL-6, as well as a range of chemokines that organize the maturation and recruitment of immune cells. In addition, IL-1 can activate the production of pro-inflammatory lipid mediators (such as prostaglandin $\mathrm{E}_{2}$, which can promote oedema ${ }^{44}$ ), induce acute phase proteins and signal to the hypothalamus to induce fever, as well as to the pituitary and adrenal glands, with direct and indirect effects on the circulatory system ${ }^{43,45,46}$.

In two independent studies, IL-1 was found to be a crucial mediator of CRS (FIG. 2). In a humanized xenograft model, blockade of IL-1-induced signalling with the IL-1R antagonist anakinra protected mice from weight loss and fever and prevented CRS-associated mortality ${ }^{33}$. In a SCID-beige xenograft model, anakinra protected mice from CRS-associated mortality and reduced macrophage expression of iNOS. CAR T cells that were engineered to overexpress IL-1Ra likewise afforded protection from the lethality of $\mathrm{CRS}^{29}$. Importantly, in both mouse models, the antitumour efficacy of the CAR T cells remained intact with concomitant suppression of CRS, when IL-1 inhibition was initiated prophylactically. Timing of treatment with anti-IL-1 therapy may be crucial for its effectiveness. Interestingly, in the humanized NSG mouse model, IL- 6 blockade did not ameliorate macrophage infiltration to the brain in contrast to IL-1R blockade ${ }^{33}$. 
Human PBMC xenograft model

An immunodeficient mouse strain with an immune system reconstituted with human peripheral blood mononuclear cells (PBMCs) that can be engrafted with human tumour tissue.
Based on these animal studies, several clinical trials have been initiated to evaluate the administration of anakinra in the context of CRS and neurotoxicity prevention (ClinicalTrials.gov NCT04148430, NCT04205838, NCT03430011, NCT04432506 and NCT04359784). Given the clinical availability of IL- $1 \beta$-blocking pharmaceuticals such as the monoclonal antibody canakinumab, future preclinical studies should shed more light on the specific roles of IL- $1 \alpha$ versus IL- $1 \beta$ in the CRS cascade, as these cytokines show large overlap in pro-inflammatory functionality but are also set apart by differences in expression and disease context ${ }^{45}$.

\section{Damage-associated molecular patterns and other soluble} mediators. The propensity of CAR T cells to induce cell death via the inflammatory process of pyroptosis rather than apoptosis results in the release of damage-associated molecular patterns such as ATP and HMGB1 (REF. $\left.{ }^{47}\right)$. The release of damage-associated molecular patterns from tumour cells can lead to the activation of macrophages in vitro to produce IL-6 and IL-1, and in vivo inhibition of pyroptosis in the SCID-beige xenograft model reduced CRS-associated mortality (but might also affect the cytolytic activity of CAR T cells $)^{47}$.

Granulocyte-macrophage colony-stimulating factor (GM-CSF) is produced by a multitude of cell types, including activated CAR T cells, and its blockade can abrogate the production of IL- 6 and other cytokines by monocytes in vitro ${ }^{48}$. However, IL- 6 production was robust and CRS eventually lethal in a xenograft model ${ }^{29}$ wherein human T cell-derived GM-CSF does not cross-react with the cognate mouse receptor ${ }^{49}$. Nevertheless, GM-CSF may still be a contributing

\section{Box 3 | Potential of IL-1 blockade in cancer immunotherapy}

Immune suppression in the tumour microenvironment (TME) and dysregulated inflammation during cytokine release syndrome (CRS) both occur in the context of immune dysfunction. Might they intersect and could IL-1 provide a link? The modulation of macrophages expressing inducible nitric oxide synthase (iNOS) ${ }^{29}$ and their reduced recruitment to the sites of chimeric antigen receptor (CAR) T cell-target interaction under certain conditions of IL-1 blockade ${ }^{137}$ build on prior evidence that the IL-1 receptor antagonist anakinra and IL-1 blockade can induce remodelling of the TME. Indeed, there are multiple reports ${ }^{138}$ that IL-1 can support tumour growth, metastasis ${ }^{139}$ and TME-mediated immunosuppression through the indirect recruitment of immunosuppressive myeloid-derived suppressor cells ${ }^{140,141}$. In a syngeneic mouse model of breast cancer, IL-1 $\beta$ deficiency resulted in reduced numbers of tumour-infiltrating myeloid-derived suppressor cells and increased proportions of mature dendritic cells, which in combination with PD1 checkpoint blockade potentiated $C D 8^{+} T$ cell-mediated antitumour immunity ${ }^{142}$. These effects were also reproduced with IL-1 $\beta$ blockade ${ }^{142}$. In a functional screen of samples derived from patients with acute myeloid leukaemia, IL-1 was found to promote the growth of myeloid progenitors in $64 \%$ of patient samples ${ }^{143}$. Furthermore, knockdown of IL-1 receptor (IL-1R1) expression in patient-derived tumour cells transplanted into mice reduced tumour outgrowth and prolonged mouse survival ${ }^{143}$. Clinical evidence strongly supports the hypothesis of IL-1 involvement in tumour incidence and progression. A fortuitous finding in the Canakinumab Anti-inflammatory Thrombosis Outcomes Study (CANTOS) trial testing the IL-1 $\beta$-blocking antibody canakinumab in the context of cardiovascular disease ${ }^{144}$ showed that IL- $1 \beta$ blockade significantly reduced the incidence of and mortality from lung cancer in smokers ${ }^{145}$. Other clinical trials in progress or completed have reported encouraging results of targeting the IL-1 pathway in various malignancies, which are reviewed elsewhere ${ }^{146}$. In summary, given the intimate involvement of the TME in both the progression of a multitude of cancers and the risks posed by CRS, it is of increased interest to combine CAR T cells with IL-1-inhibiting therapies ${ }^{116}$. factor in CRS without being a requirement for it. In a human PBMC xenograft model, blockade of both mouse and human GM-CSF decreased IL-6 production ${ }^{50}$ (FIGS 2,3).

Tumour necrosis factor (TNF) is another pleiotropic cytokine that mediates inflammation through the activation of T cells, macrophages, monocytes and other cells of the immune system. In contrast to IL-1 and IL-6, TNF also has direct tumoricidal activity ${ }^{51}$. TNF was recently proposed as a potential contributor to CRS induced by CD3 and HER2 bispecific antibodies in a HER2-humanized mouse mammary tumour virus (MMTV) model of breast cancer. Pretreatment with TNF inhibitors in this model reduced circulating IL-6 levels, and in some cases IL-1 levels, but did not impair antitumour efficacy ${ }^{52}$. In the SCID-beige xenograft model $^{29}$, TNF blockade significantly reduced IL- 6 production by myeloid cells and abrogated CRS-associated mortality, but could also reduce the antitumour activity of CAR T cells depending on the CAR construct (T.G., unpublished data) (FICS 2,3).

Interferon- $\gamma$ (IFN $\gamma)$ is abundantly produced by activated $\mathrm{T}$ cells and can promote macrophage maturation and enhance NO production through iNOS induction $^{53,54}$. Moreover, IFN $\gamma$ can promote increased permeabilization of other tissues, including the BBB, by loosening tight junctions ${ }^{49-51,55-58}$. In patients with severe CRS, the serum concentration of IFN $\gamma$ can be increased up to 100-fold, as is also observed in macrophage activation syndrome (BOX 1). However, despite this observation, no specific pathogenic role for IFN $\gamma$ in CRS has yet been described.

The adrenal system is also implicated in the development and maintenance of CRS, as the catecholamines adrenaline and noradrenaline directly influence the activation of CAR T cells and subsequent cytokine release. Administration of atrial natriuretic peptide (ANP), a regulator of electrolytes and extracellular fluid volume and an inhibitor of cytokine secretion ${ }^{59}$, or inhibition of catecholamine synthesis by metirosine diminishes cytokine production by CAR T cells in vitro and in vivo, and also reduces mortality in a xenograft mouse model ${ }^{60}$ (FIGS 2,3). In a syngeneic mouse model of B cell acute lymphoblastic leukaemia ${ }^{61}$, metirosine at a lower dose did not impair antitumour efficacy. Interestingly, immune cells were reported not only to be activated by catecholamines but also to natively produce catecholamines upon activation, and therefore create a positive-feedback loop ${ }^{61}$. However, dysregulated levels of ANP are known to promote oedema, decreased blood pressure and electrolyte imbalances, all of which are common in CRS pathology. The safety and feasibility of blocking catecholamine receptors, especially at the onset of CRS, would thus raise some concerns.

\section{Pathophysiology of ICANS}

Despite the clinical features of ICANS being readily recognizable, its pathophysiology remains poorly understood. Recent animal models have implicated endothelial cell activation and disruption of the $\mathrm{BBB}$ resulting in direct neuronal cell injury, in addition to a role for various pro-inflammatory cytokines. However, these models 


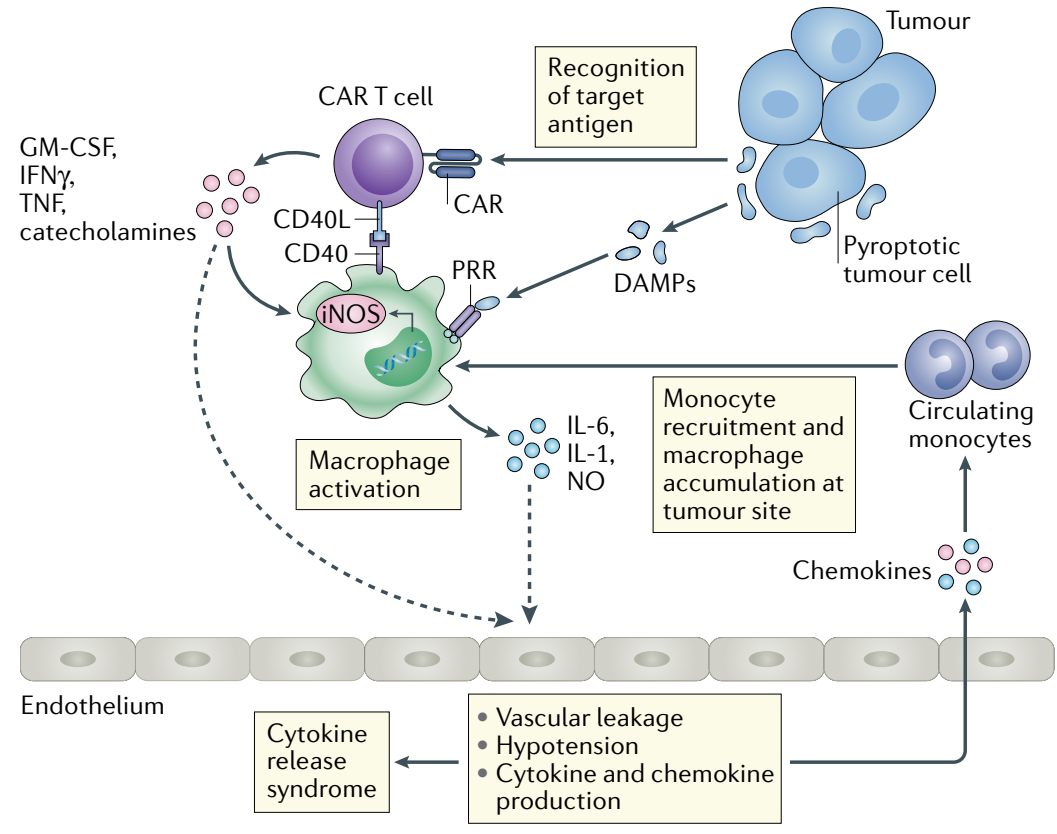

Fig. 2 | Working model of the pathophysiological mechanisms of CRS. Upon target recognition, chimeric antigen receptor (CAR) T cells are activated to produce cytokines, such as interferon- $\gamma$ (IFN $\gamma$ ), granulocyte-macrophage colony-stimulating factor (GM-CSF) and tumour necrosis factor (TNF), and soluble inflammatory mediators (for example, catecholamines) that can activate macrophages and surrounding tissues. In addition, damage-associated molecular patterns (DAMPs) released by pyroptotic tumour cells are recognized by pattern-recognition receptors (PRRs) on macrophages and can further amplify their activation. In a contact-dependent manner, macrophage-expressed CD40 can be engaged by CAR T cell-expressed CD40 ligand (CD40L) and promote macrophage activation. Activated macrophages secrete inflammatory mediators, among which IL-6, IL-1 and nitric oxide (NO) produced by inducible nitric oxide synthase (iNOS) have been shown to be directly involved in the pathology of cytokine release syndrome (CRS). These cytokines may drive the systemic pathology of CRS owing to their ability to signal to a range of non-immune tissues such as the endothelium, which can result in vascular leakage, hypotension and further amplification of the inflammatory response by the secretion of cytokines and chemokines. It is hypothesized that locally produced chemokines attract circulating monocytes, which leads to an accumulation of activated macrophages at the site of interaction between CAR T cells and tumour cells, thus amplifying the inflammatory loop. Dashed lines denote hypothesized pathways that have not been experimentally confirmed in the context of CRS.

of CAR T cell-mediated neurotoxicity are limited by an absence of human cytokines and haematopoietic cells, together with the occurrence of concomitant xenograft versus host disease ${ }^{33,62-64}$. Despite this, recent research in mice and non-human primates has generated important insights, which, together with data from clinical trials of patients developing ICANS after infusion of CAR T cells, have improved our understanding of the pathophysiology of ICANS (FIG. 4).

Vascular permeability, endothelial disruption and glial cell injury. Patients with ICANS have increased levels of protein, $\mathrm{CD} 4^{+} \mathrm{T}$ cells, $\mathrm{CD} 8^{+} \mathrm{T}$ cells and CAR $\mathrm{T}$ cells in the CSF, which indicates loss of integrity of the BBB. Clinical studies have shown an association between the number of CAR T cells and level of cytokines in the CSF and the severity of ICANS ${ }^{17,21,65}$.

Some biochemical features of severe CRS and ICANS that are observed in patients, such as hypofibrinogenaemia and increased fibrin degradation products, are shared with features of disseminated intravascular coagulation and endothelial cell disruption, which are typically seen in sepsis and critical illness associated with increased vascular permeability ${ }^{66}$. Indeed, there is evidence of vascular leak in patients with severe ICANS. Changes in the angiopoietin (ANG)-TIE2 axis, which in health regulates endothelial cell activation ${ }^{67}$, have been described in a single-centre study of 133 patients treated with CD19CAR T cells ${ }^{16}$. ANG1 is constitutively produced by platelets and perivascular cells, and when bound to its endothelial receptor, TIE2, stabilizes the endothelium. Following endothelial cell activation by inflammatory cytokines, ANG2 is released from endothelial Weibel-Palade bodies and displaces ANG1, which further increases endothelial cell activation and microvascular permeability. Consistent with this mechanism, statistically significant increased ratios of serum ANG2 to ANG1 were observed in patients with severe ICANS compared with those with less severe neurotoxicity, together with higher concentrations of von Willebrand factor (vWF) and CXC-chemokine ligand 8 (CXCL8), all of which are produced by platelets and perivascular cells $^{68}$. In addition, patients with increased serum ANG2 to ANG1 ratios prior to lymphodepletion and CAR $\mathrm{T}$ cell administration had a higher risk of developing ICANS. Further data point towards the sequestration of high molecular weight vWF multimers in patients with severe ICANS, resulting in coagulopathy. However, the factors that control baseline and perturbed levels of ANG2 and ANG1 remain elusive.

In addition to disruption of the BBB and increased vascular permeability, glial cell injury has been reported in paediatric and young adult patients who develop ICANS following CD19CAR T cell therapy ${ }^{69}$. In a cohort of 43 patients, a significant increase in GFAP and S100b levels in the CSF was found in patients with acute neurotoxicity. GFAP is a well-validated marker of astroglial cell injury irrespective of cause ${ }^{70}$, whereas S100b in the CSF is a marker of astrocyte activation ${ }^{71}$. In this same study, increased levels of IL-6, IL-10, IFN $\gamma$ and granzyme B in CSF were also found to be associated with neurotoxicity.

Cytokines and their cell sources. In the humanized NSG mouse model ${ }^{33}$, the infusion of human CD19CAR T cells bearing either CD28 or 4-1BB co-stimulatory domains resulted in B cell aplasia, CRS and neurotoxicity. These mice developed delayed fatal ICANS following initial CRS, a pattern that is also observed in some patients (typically $<1 \%$ of them $)^{14,72}$. However, in this model, blockade of signalling through IL-6R had no impact on neurotoxicity. By contrast, IL-1R blockade eliminated both CRS and neurotoxicity without affecting CAR T cell efficacy. Monocyte ablation had a negative effect on CAR T cell proliferation and population expansion. These differences might be explained by the fact that the IL-1R antagonist anakinra is known to cross the $\mathrm{BBB}^{73,74}$, whereas there is no clear evidence that the IL-6R-specific monoclonal antibody tocilizumab can penetrate the CNS.

A non-human primate model of ICANS using immunocompetent rhesus macaques has recently been reported ${ }^{74}$. These primates developed features consistent 
Conditioning therapy

Chemotherapy and/or radiotherapy delivered prior to the infusion of chimeric antigen receptor (CAR) T cells, with the aim of increasing the expansion of the infused T cell population.

with ICANS following the infusion of CD20CAR T cells bearing a 4-1BB co-stimulatory domain. Although both CAR T cells and non-CAR T cells were observed to be increased in number in the CSF and brain parenchyma during peak neurotoxicity, the number of CAR $\mathrm{T}$ cells was proportionately higher than the number of non-CAR T cells. This was associated with high concentrations of IL-6, CXCL8, IL-1Ra, CXCL9, CXCL11, GM-CSF and vascular endothelial growth factor, which were found at higher levels in CSF than in the corresponding serum samples. Histological panencephalitis, including multifocal meningitis and perivascular $\mathrm{T}$ cell infiltration, was observed to varying degrees 8 days after CAR T cell infusion, which coincided with peak levels of CAR T cell proliferation in the peripheral blood. Detailed phenotypic analyses identified a significant increase in cell surface expression of the integrin VLA4 by CAR T cells, compared with non-CAR T cells, which may have facilitated increased trafficking of CAR T cells into the CNS. Taken together, these findings suggest that the mechanisms driving ICANS may include accumulation of both pro-inflammatory cytokines and CAR $\mathrm{T}$ cells in the CNS, although their relative contributions are so far unknown.

Numerous clinical trials have shown an association between increased serum levels of various cytokines and the risk of developing ICANS ${ }^{10,13,16,17}$. Those cytokines that are consistently increased in patients in multiple studies (using various CAR constructs and with different target malignancies) include IL-2, IL-6, IL-10 and IL-15. However, whereas mouse models have suggested a clear role for recipient monocyte-derived immune cells in the secretion of cytokines and pathogenesis of CRS and ICANS, in clinical trials it is not possible to identify the specific cell source of cytokines in patients who develop ICANS. Nevertheless, a marked increase in the number

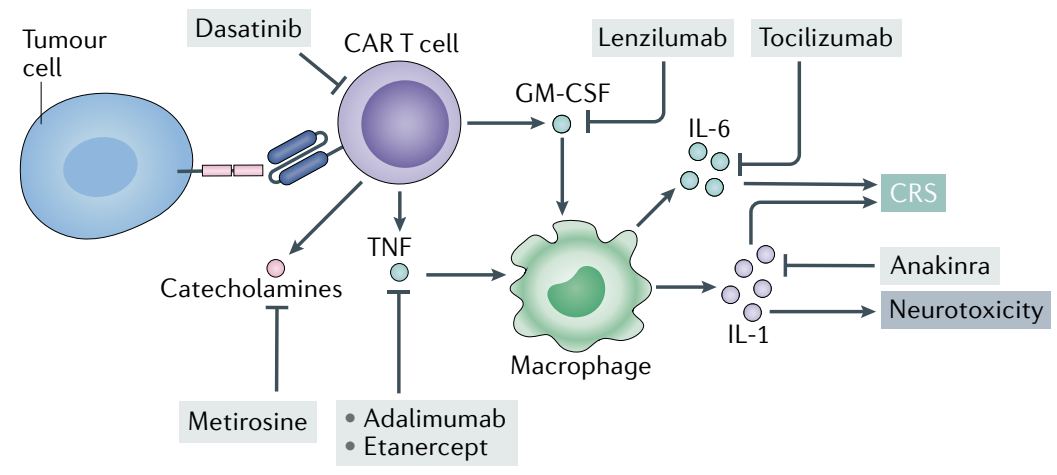

Fig. 3 | Schematic representation of current and potential therapeutic interventions for CRS. Blockade of the IL-6 receptor (IL-6R; using the monoclonal antibody tocilizumab) has shown clinical benefit in patients with cytokine release syndrome (CRS) , $, 6,29,33^{\text {. }}$. In vivo and in vitro models have proposed novel therapeutic interventions for CRS that directly target pro-inflammatory cytokines - such as IL-1 (the IL-1 receptor antagonist

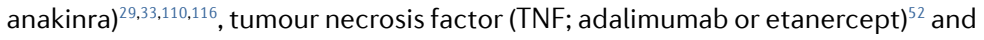
granulocyte-macrophage colony-stimulating factor (GM-CSF; lenzilumab) ${ }^{48,50}$ —or other pro-inflammatory mediators such as catecholamines (for example metirosine, which inhibits catecholamine synthesis ${ }^{60}$. Furthermore, kinase inhibitors such as dasatinib can inhibit chimeric antigen receptor (CAR) T cell functionality, with subsequent reduction in effector cytokine secretion ${ }^{108}$. Other broad-spectrum small-molecule inhibitors, such as ruxolitinib and ibrutinib (not shown here), that can broadly inhibit cytokine signalling and cytokine production across multiple cell types have been proposed for use in CRS. of myeloid cells has been observed in the CSF of patients who develop severe ICANS ${ }^{66}$.

Target antigen expression in the CNS. Data from clinical trials have shown that the presence of antigen-positive tumour cells in the CNS is not required for the development of ICANS ${ }^{66}$. Moreover, it is noteworthy that when CAR T cells are infused intrathecally or intratumourally in patients with glioblastoma multiforme, these patients do not develop ICANS. However, in a recent study, single-cell RNA-sequencing analyses demonstrated CD19 expression in human brain mural cells, including pericytes and vascular smooth muscle cells, which raises the possibility that an on-target off-tumour effect may contribute to the neurotoxicity associated with CD19CAR T cells ${ }^{75}$. This observation could explain the higher incidence of ICANS observed with CD19-directed therapies compared with those targeting CD20, CD22 and BCMA (also known as TNFRSF17), although alternate or additional mechanisms may apply. CD22, which is expressed by microglia in human brain $^{76,77}$, has not been associated with a higher incidence or severity of ICANS in the setting of CD22CAR T cell therapy. Microglia are specialized phagocytic cells that persist in the CNS for decades. They phagocytose myelin debris and protein aggregates, thus preventing damage to neuronal cells and preserving brain homeostasis and function $^{78-80}$. A previously undescribed role for CD22 as a negative regulator of microglial phagocytosis has been identified using a CRISPR-Cas9 knockout screen in combination with RNA sequencing ${ }^{80}$. In aged mice with ageing microglia, CD22 was upregulated, which impaired the clearance of myelin debris, $\beta$-amyloid oligomers and $\alpha$-synuclein fibrils in vivo. Administration of CD22-blocking antibodies reversed microglial dysfunction and improved phagocytosis and cognitive function. The role of microglial phagocytosis in the pathogenesis of ICANS and the consequences of CD22 expression by microglia for CD22CAR $\mathrm{T}$ cell therapy are yet to be elucidated, but cytokine-mediated activation of microglial cells has been described in children with cerebral malaria ${ }^{76}$. These children develop a diffuse encephalopathy with disruption of the BBB and cerebral oedema, clinical features that are not dissimilar to those of ICANS.

Cerebral oedema. Cerebral oedema is a rare but potentially fatal neurological complication that has been observed after CAR T cell therapy ${ }^{16,65,81}$. Available evidence suggests that the pathophysiology of cerebral oedema may be different from that of the more common manifestation of encephalopathy seen with ICANS. In one clinical trial, evaluating CD19CAR T cells in adult patients with B cell acute lymphoblastic leukaemia, five patients developed fatal cerebral oedema, leading to termination of the trial ${ }^{81}$. A root cause analysis evaluating patient characteristics, conditioning therapy and product attributes showed that the patients who developed cerebral oedema were younger than 30 years of age, had a higher percentage of $\mathrm{CD}^{+} \mathrm{T}$ cells in the CAR T cell product, had higher serum IL-15 levels and low levels of blood platelets prior to CAR $\mathrm{T}$ cell infusion, and had rapid 


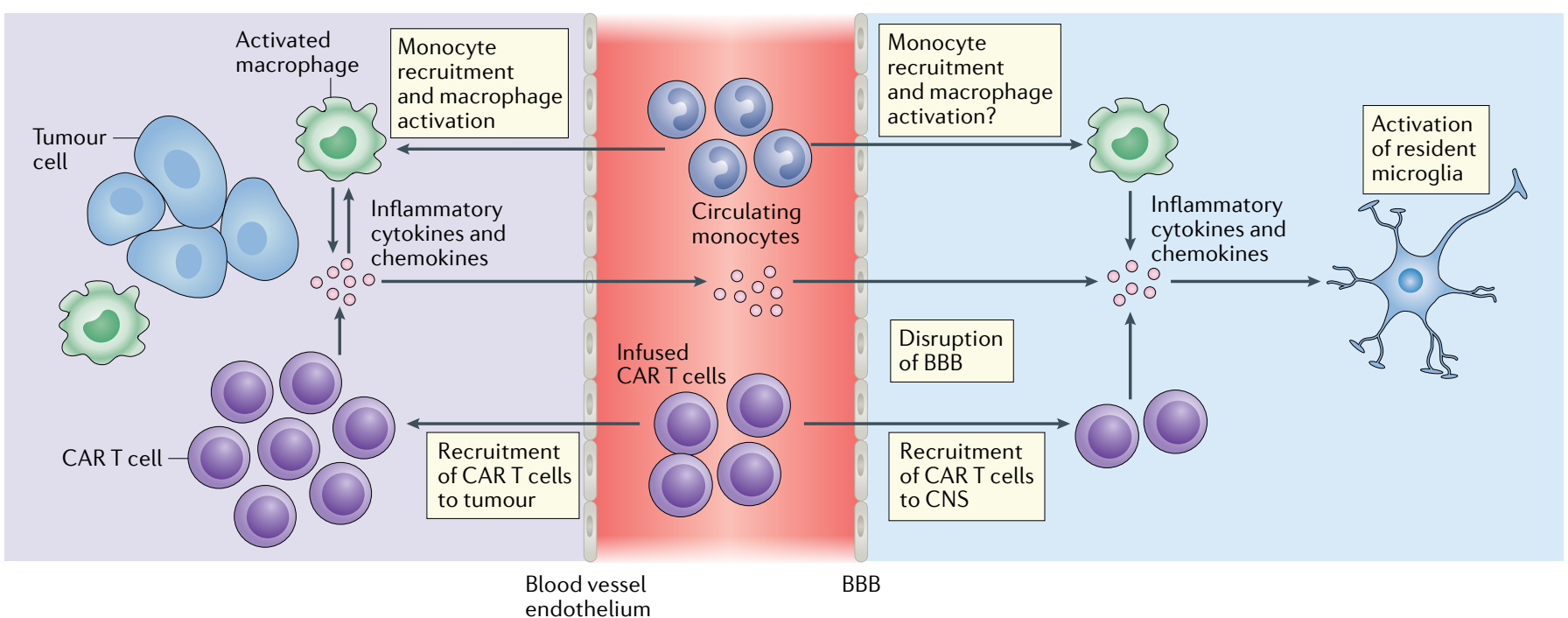

Fig. 4 | Pathophysiology of ICANS. Similar to cytokine release syndrome (CRS), the pathophysiology of immune effector cell-associated neurotoxicity syndrome (ICANS) seems to start with the production of pro-inflammatory cytokines by chimeric antigen receptor (CAR) T cells and the activation of bystander immune cells such as macrophages in the tumour microenvironment. Inflammatory cytokines and chemokines produced by CAR T cells and myeloid cells in the tumour microenvironment - such as IL-1 $\beta$, IL-6, IL-10, the chemokines CXCL8 and CCL2, interferon- $\gamma$, granulocytemacrophage colony-stimulating factor and tumour necrosis factor - diffuse into the bloodstream and, eventually, result in disruption of the blood-brain barrier (BBB), with accumulation of cytokines and CAR T cells in the central nervous system (CNS) together with activation of resident microglial cells.

expansion of CAR T cell populations peaking within the first week, which was associated with a sharp increase in serum levels of IL-2 and TNF ${ }^{81}$. Importantly, autopsies on two patients revealed complete breakdown of the BBB but an absence of activated T cells in the CNS. Although they are not definitive, these results suggest that the breakdown of the BBB and subsequent cerebral oedema were probably owing to the inflammatory cytokine surge rather than to CAR T cell infiltration into the CNS. Moreover, the analysis indicated that cerebral oedema could result from a combination of factors that include both patient characteristics and product attributes.

\section{Clinical management of CRS and ICANS}

Low-grade CRS is managed by supportive care with antipyretics while ensuring that there is no concurrent cause for fever, such as infection. Moderate to severe CRS is treated with the IL-6R-blocking antibody tocilizumab with or without immunosuppression with corticosteroids, together with intensive supportive care including fluid resuscitation and vasopressors for hypotension and supplemental oxygen delivery as needed for hypoxia ${ }^{9,14,82-84}$. Low-grade ICANS is also typically managed by diagnostic work-up and supportive care, whereas severe ICANS is usually treated with corticosteroids at most centres ${ }^{14,84}$. The use of tocilizumab has markedly reduced the incidence of severe CRS, likely owing to the fact that levels of IL-6 peak early during CRS and it is a key mediator of the downstream inflammatory cascade 4 . However, in very severe cases of CRS, corticosteroids are also required for management; multiple cytokines might have a role in the later stages of $\mathrm{CRS}^{13}$ and corticosteroids can induce global immune suppression, including inhibiting the proliferation of and cytokine secretion by CAR T cells and other bystander immune cells, in particular myeloid cells.

Whereas tocilizumab is very effective for the management of CRS, it has no effect in most cases of ICANS $^{14,16,17,84}$. This may be because of the differences in pathophysiology between CRS and ICANS and/or poor penetration of tocilizumab across the BBB. Indeed, prophylactic use of tocilizumab decreased the incidence of severe CRS but increased the occurrence of severe ICANS, likely owing to the increase in serum IL-6 levels observed after tocilizumab administration that results from preventing its uptake into peripheral tissues by receptor blockade ${ }^{65,85}$. However, these observations need to be interpreted with caution as the study was not randomized and the sample size was small ${ }^{65}$. Although corticosteroids can cross the BBB and are commonly used for the management of ICANS ${ }^{10,86}$, definitive evidence for their clinical benefit on the severity or duration of ICANS is lacking. Most studies indicate that the use of tocilizumab does not seem to affect the efficacy of CAR $\mathrm{T}$ cells ${ }^{10,15,86}$. However, the data on the effects of corticosteroids on CAR T cell efficacy are conflicting, with some studies indicating no impact but others showing adverse clinical outcomes with increased risk of early progression and death ${ }^{10,87,88}$. This further highlights the need for better understanding of the pathophysiological mechanisms of CRS and ICANS and the development of novel strategies for their management.

Effects of other clinical factors. The onset, severity and duration of CRS and ICANS after CAR T cell therapy may be affected by factors related to the host, tumour 
and/or therapy. Patients with a higher baseline inflammatory state - as defined by levels of C-reactive protein, ferritin, D-dimer and pro-inflammatory cytokines - have increased risk of developing CRS and ICANS ${ }^{89,90}$. It is possible that these patients have a predisposition to a greater inflammatory response after CAR T cell infusion. More severe cases of CRS and/or ICANS have also been associated with higher tumour burden in multiple malignancies including leukaemia, lymphoma and multiple myeloma, which is likely to be associated with greater expansion of CAR T cell populations and synchronous activation ${ }^{13,31,86,91-93}$. The intensity of the conditioning therapy before CAR T cell infusion also seems to impact the severity of these toxicities, with more intense regimens increasing the risk of severe CRS and/ or ICANS, probably by inducing greater lymphodepletion, which eliminates cytokine sinks and makes higher levels of homeostatic cytokines, such as IL-2 and IL-15, available for CAR T cell proliferation ${ }^{5,94-96}$. Interestingly, older age was not associated with increased risk of severe CRS or ICANS $86,90,97$.

Effects of CAR design. Recent studies indicate that the design of the CAR molecule itself could significantly affect the proliferation and cytokine profile of the CAR $\mathrm{T}$ cells, and thereby influence the incidence and severity of CRS and/or ICANS. As discussed above, CAR $\mathrm{T}$ cell products with a CD28 co-stimulatory signalling domain seem to proliferate more rapidly after infusion and their numbers seem to peak earlier than products engineered with the 4-1BB co-stimulatory signalling domain. In clinical studies, this has been associated with earlier onset and higher incidence of more severe CRS and ICANS ${ }^{10,15}$. However, a direct comparison of CAR $T$ cell products with CD28 versus 4 - $1 \mathrm{BB}$ co-stimulatory domains has not been carried out so far. It is possible that differences in patient characteristics such as the tumour burden and variation in monitoring and grading of toxicities between studies could account for some of the reported differences between CD28 and 4-1BB domains in terms of both pharmacokinetics and adverse events.

Evidence in the literature also indicates that altering the non-signalling domains of the CAR molecule, including hinge and transmembrane regions or the antigen-binding domain ( $\mathrm{scFv}$ ), may impact toxicities associated with CAR T cell therapy. For example, altering the length of the CD8a hinge and transmembrane domain decreased the cytokine production of CD19CAR T cells bearing $4-1 \mathrm{BB}$ and $\mathrm{CD} 3 \zeta$ signalling domains and reduced their proliferation, but retained their cytolytic activity, in preclinical models and in a phase I clinical trial ${ }^{98}$. Importantly, testing this product in patients with $\mathrm{B}$ cell lymphoma resulted in a high rate of complete response with only low-grade CRS and no ICANS ${ }^{98}$. CD19CAR T cells with a low-affinity scFv have high antitumour efficacy but without inducing severe CRS in paediatric patients with acute lymphoblastic leukaemia, compared with published studies using tisagenlecleucel, a CD19CAR T cell therapy that uses a high-affinity $\mathrm{scFv}^{11,99}$. Patients with B cell lymphoma who were treated with CD19CAR T cells that contain CD28 and
$\mathrm{CD} 3 \zeta$ signalling domains identical to the approved CD19CAR T cell therapy axicabtagene ciloleucel $^{95,100}$, but have different $s c F v$ and hinge and transmembrane domains, had similar anti-lymphoma activity but much lower incidence and severity of ICANS ${ }^{101}$. In a small study testing CD19CAR natural killer cells, high rates of complete response were noted in patients with $\mathrm{B}$ cell malignancies without the induction of CRS or ICANS, which suggests that different immune effector cells may have different toxicity profiles ${ }^{102}$. Lastly, CARs encoding a single immunoreceptor tyrosine-based activation motif in $\mathrm{CD} 3 \zeta$ (rather than three of these motifs) have been shown in preclinical studies to enhance therapeutic efficacy and memory $\mathrm{T}$ cell differentiation without increasing inflammatory activity ${ }^{103}$.

Collectively, these reports indicate that reducing the tumour burden and the baseline inflammatory state, adjusting the conditioning regimen and optimizing the design of the CAR molecule and/or the CAR T cell product may reduce the incidence and/or severity of CRS and ICANS.

\section{New strategies to manage CRS and ICANS}

Cytokine hypersecretion and dysregulation are central to the pathology of CRS. Non-specific immunosuppressive agents such as corticosteroids relieve patient symptoms in many cases ${ }^{4,7,12,27,104}$. Moreover, other broad-spectrum cytokine inhibitors - such as ruxolitinib, which blocks JAK1 and JAK2, or itacitinib, which blocks JAK1 (kinases that are required for cytokine receptor signalling) would be expected to blunt the effects of pro-inflammatory cytokines such as IFN $\gamma$ and IL-6 (REFS ${ }^{37,105}$ ). Indeed, in preclinical models of CAR T cell-related toxicity, ruxolitinib and itacitinib reduced toxicity and cytokine secretion $^{105}$. Similarly, ibrutinib, a Bruton's tyrosine kinase (BTK) inhibitor that also inhibits IL-2 tyrosine kinase (ITK; a kinase involved in proximal T cell receptor signalling $)^{106}$, resulted in reduced cytokine secretion in vivo in a mouse model, including secretion of IL-6, but also led to a reduction in levels of CAR T cell-derived cytokines such as IFN $\gamma$, which could indicate blunted CAR T cell activation. In this study, antitumour efficacy was monitored only in the short term (day 4 after CAR T cell transfer) with no observed differences ${ }^{107}$. Interestingly, in co-culture with a mantle-cell lymphoma cell line, ibrutinib reduced cytokine secretion of tumour cells, demonstrating the broad effect of such treatment approaches. Small-molecule kinase inhibitors can often engage multiple targets. These results raise the possibility that ruxolitinib and ibrutinib could directly affect CAR $\mathrm{T}$ cell activation levels and, thus, influence clinical outcomes.

Another study used the incomplete specificity of kinase inhibitors to create an on/off switch for CD3 $\zeta$ chain-based CAR T cells ${ }^{108}$. Dasatinib, a BCR-ABLtargeting kinase inhibitor that is approved for the treatment of various haematological malignancies, was shown to potently inhibit CAR T cell-mediated cytotoxicity and cytokine production in a rapid and reversible manner. Moreover, short-term dosing of dasatinib in preclinical models reduced CRS-associated mortality 
without impairing in vivo antitumour efficacy upon removal of the drug and resumption of CAR $\mathrm{T}$ cell activity $^{108}$ (FIG. 3). Further supporting an integral role for activated CAR T cells in initiating CRS, another preclinical study recently showed that attenuation of LCK signalling through the engineered recruitment of the phosphatase SHP1 to the immunological synapse can reduce the production of effector cytokines by CAR T cells and CRS severity ${ }^{109}$. In addition, a BBz CAR (bearing 4-1BB and $\mathrm{CD} 3 \zeta$ co-stimulatory domains) with a modified architecture of its hinge, transmembrane and membrane-proximal intracellular domains had a phenotype of blunted activation and reduced effector cytokine production, resulting in decreased toxicity preclinically ${ }^{98}$ in the SCID-beige xenograft model ${ }^{30}$ and in the clinic when compared with historical data ${ }^{98}$.

Cumulatively, these studies suggest that broadspectrum cytokine inhibition through different mechanisms can reduce CRS pathology, an outcome that is expected given what we know about the pathophysiological mechanisms of CRS. Nonetheless, the long-term inhibition of cytokines may be detrimental to the antitumour efficacy of CAR T cells and, for this reason, targeted interventions aiming to selectively disrupt specific cytokine signalling pathways may be favoured. In a small case series of eight patients with severe ICANS or haemophagocytic lymphohistiocytosis following CD19CAR T cell therapy, IL-1R blockade with anakinra seemed to provide benefit in half of the patients ${ }^{110}$. The judicious use of novel agents targeting additional cytokines such as IFN $\gamma$ (for example, emapalumab) may help to elucidate their role in the management of severe CRS ${ }^{111}$. Future clinical studies should be able to demonstrate the benefits of such approaches when used in prophylactic and/or therapeutic settings.

\section{Conclusions and future directions}

Currently, the widespread adoption of CAR T cell therapies is limited, in part, by the requirement for treatment in centres that are experienced in managing the common toxicities of CRS and ICANS and by the financial and health burden that this creates. A greater understanding of the molecular and cellular pathophysiologies of CRS and ICANS will facilitate the development of effective targeted therapies with reduced toxicities without compromising antitumour activity. Already, novel CAR constructs are being designed to minimize the risk of eliciting CRS and ICANS, while optimizing recognition of tumour antigen and effective T cell signalling. Another current limitation is the need for a greater understanding of the biology and mechanisms of action of CAR T cells. This includes the impact of the biophysical properties of the CAR and its co-stimulatory domain on gene expression profiles, which may alter $\mathrm{T}$ cell subset, function, memory potential and exhaustion. To improve the clinical efficacy of CAR T cell therapies, there is an urgent requirement to generate $\mathrm{T}$ cells with optimal in vivo fitness, and durable persistence and efficacy. Beyond oncology, in the fields of autoimmunity and solid organ transplantation, there is great interest in using CAR-based technologies for the generation of antigen-specific regulatory $\mathrm{T}$ cells, which have the potential to deliver targeted immunosuppression ${ }^{112-115}$. The in vivo biology and function of these regulatory CAR T cells will differ from those properties observed so far for effector CAR T cells, and some novel toxicities may also occur. The safe and broad use of engineered T cell therapies in oncology and for non-malignant indications will depend on achieving effective and dependable prevention of these complications.

Published online 17 May 2021
1. June, C. H. \& Sadelain, M. Chimeric antigen receptor therapy. N. Engl. J. Med. 379, 64-73 (2018)

Brentjens, R. J. et al. CD19-targeted T cells rapidly induce molecular remissions in adults with chemotherapy-refractory acute lymphoblastic leukemia. Sci. Transl. Med. 5, 177 ra38 (2013).

3. Grupp, S. A. et al. Chimeric antigen receptor-modified $\mathrm{T}$ cells for acute lymphoid leukemia. N. Engl. J. Med. 368, 1509-1518 (2013).

Together with Brentjens et al. (2013), this article reports early clinical data demonstrating the efficacy of CD19CAR T cells in patients with refractory acute lymphoblastic leukaemia.

4. Maude, S. L. et al. Chimeric antigen receptor T cells for sustained remissions in leukemia. N. Engl. J. Med. 371, 1507-1517 (2014).

5. Kochenderfer, J. N. et al. Chemotherapy-refractory diffuse large B-cell lymphoma and indolent B-cell malignancies can be effectively treated with autologous T cells expressing an anti-CD 19 chimeric antigen receptor. J. Clin. Oncol. 33, 540-549 (2015)

6. Davila, M. L. et al. Efficacy and toxicity management of 19-28z CAR T cell therapy in B cell acute lymphoblastic leukemia. Sci. Transl. Med. 6, 224 ra25 (2014).

7. Maher, J. et al. Human T-lymphocyte cytotoxicity and proliferation directed by a single chimeric TCR /CD28 receptor. Nat. Biotechnol. 20, 70-75 (2002).

This article is the first demonstration that fusion receptors containing both $\mathrm{T}$ cell receptor and CD28 signalling moieties could redirect and amplify human $\mathrm{T}$ cell responses.

8. Imai, C. et al. Chimeric receptors with 4-1BB signaling capacity provoke potent cytotoxicity against acute lymphoblastic leukemia. Leukemia. 18, 676-684 (2004).
9. Lee, D. W. et al. Current concepts in the diagnosis and management of cytokine release syndrome. Blood 124, 188-195 (2014).

10. Neelapu, S. S. et al. Axicabtagene ciloleucel CAR T-cell therapy in refractory large B-cell lymphoma. N. Engl. J. Med. 377, 2531-2544 (2017).

11. Maude, S. L. et al. Tisagenlecleucel in children and young adults with B-cell lymphoblastic leukemia. N. Engl. J. Med. 378, 439-448 (2018).

12. Brudno, J. N. \& Kochenderfer, J. N. Toxicities of chimeric antigen receptor $\mathrm{T}$ cells: recognition and management. Blood 127, 3321-3330 (2016).

13. Hay, K. A. et al. Kinetics and biomarkers of severe cytokine release syndrome after CD19 chimeric antigen receptor-modified T-cell therapy. Blood 130 , 2295-2306 (2017).

14. Neelapu, S. S. et al. Chimeric antigen receptor T-cell therapy - assessment and management of toxicities. Nat. Rev. Clin. Oncol. 15, 47-62 (2018).

This review discusses clinical aspects of CAR T cell-mediated toxicities

15. Schuster, S. J. et al. Tisagenlecleucel in adult relapsed or refractory diffuse large B-cell lymphoma. N. Engl. J. Med. 380, 45-56 (2019).

16. Gust, J. et al. Endothelial activation and blood-brain barrier disruption in neurotoxicity after adoptive immunotherapy with CD 19 CAR-T cells. Cancer Discov. 7, 1404-1419 (2017).

17. Santomasso, B. D. et al. Clinical and biological correlates of neurotoxicity associated with CAR T-cell therapy in patients with B-cell acute lymphoblastic leukemia. Cancer Discov. 8, 958-971 (2018).

18. Locke, F. L., Go, W. Y. \& Neelapu, S. S. Development and use of the anti-CD19 chimeric antigen receptor T-cell therapy axicabtagene ciloleucel in large B-cell lymphoma: a review. JAMA Oncol. 6, 281-290 (2019).

19. Brentjens, R. J. et al. Eradication of systemic B-cell tumors by genetically targeted human T lymphocytes co-stimulated by CD80 and interleukin-15. Nat. Med. 9, 279-286 (2003).

20. Kowolik, C. M. et al CD28 costimulation provided through a CD19-specific chimeric antigen receptor enhances in vivo persistence and antitumor efficacy of adoptively transferred T cells. Cancer Res. 66, 10995-11004 (2006).

21. Lee, D. W. et al. T cells expressing CD19 chimeric antigen receptors for acute lymphoblastic leukaemia in children and young adults: a phase 1 dose-escalation trial. Lancet 385, 517-528 (2015).

22. Pennisi, M. et al. Comparing CAR T-cell toxicity grading systems: application of the ASTCT grading system and implications for management. Blood $A d v$. 4, 676-686 (2020).

23. Le, R. Q. et al. FDA approval summary: tocilizumab for treatment of chimeric antigen receptor $\mathrm{T}$ cell-induced severe or life-threatening cytokine release syndrome. Oncologist 23, 943-947 (2018).

24. van der Stegen, S. J. C. et al. Preclinical in vivo modeling of cytokine release syndrome induced by ErbB-retargeted human T cells: identifying a window of therapeutic opportunity? J. Immunol. 191 4589-4598 (2013).

25. Teachey, D. T. et al. Identification of predictive biomarkers for cytokine release syndrome after chimeric antigen receptor T-cell therapy for acute lymphoblastic leukemia. Cancer Discov. 6, 664-679 (2016).

26. Zhao, Z. et al. Structural design of engineered costimulation determines tumor rejection kinetics and persistence of CAR T cells. Cancer Cell 28, 415-428 (2015).

27. Oluwole, O. O. \& Davila, M. L. At the bedside: clinical review of chimeric antigen receptor (CAR) T cell therapy for B cell malignancies. J. Leukoc. Biol. 100, 1265-1272 (2016). 
28. Shimabukuro-Vornhagen, A. et al. Cytokine release syndrome. J. Immunother Cancer 6, 56 (2018).

29. Giavridis, T. et al. CAR T cell-induced cytokine release syndrome is mediated by macrophages and abated by IL-1 blockade. Nat. Med. 24, 731-738 (2018). This study reports the role of recipient macrophage-derived IL-1, IL-6 and NO in the pathogenesis of CRS in a mouse model.

30. Fry, T. J. et al. CD22-targeted CAR T cells induce remission in B-ALL that is naive or resistant to CD19-targeted CAR immunotherapy. Nat. Med. 24, 20-28 (2018).

31. Shah, N. N. et al. CD4/CD8 T-cell selection affects chimeric antigen receptor (CAR) T-cell potency and toxicity: updated results from a phase I anti-CD22 CAR T-cell trial. J. Clin. Oncol. 38, 1938-1950 (2020).

32. Baird, J. H. et al. CD22-directed CAR T-cell therapy induces complete remissions in CD19-directed CAR-refractory large B-cell lymphoma. Blood https://doi.org/10.1182/blood.2020009432 (2020).

33. Norelli, M. et al. Monocyte-derived IL- 1 and IL- 6 are differentially required for cytokine-release syndrome and neurotoxicity due to CAR T cells. Nat. Med. 24 739-748 (2018)

This study in humanized mice identifies the roles of monocyte-derived IL-1 and IL- 6 as key cytokines mediating CRS and ICANS.

34. Nijmeijer, B. A., Willemze, R. \& Falkenburg, J. H. F. An animal model for human cellular immunotherapy: specific eradication of human acute lymphoblastic leukemia by cytotoxic T lymphocytes in NOD/SCID mice. Blood 100, 654-660 (2002)

35. Park, J. H. et al. Long-term follow-up of CD 19 CAR therapy in acute lymphoblastic leukemia. N. Engl. J. Med. 378, 449-459 (2018)

36. Nashleanas, M. \& Scott, P. Activated T cells induce macrophages to produce NO and control Leishmania major in the absence of tumor necrosis factor receptor p55. Infect. Immun. 68, 1428-1434 (2000).

37. Orabona, C. et al. CD28 induces immunostimulatory signals in dendritic cells via CD80 and CD86. Nat. Immunol. 5, 1134-1142 (2004). This mouse study describes the crucial role of CD80- and CD86-mediated co-stimulation in $\mathrm{T}$ cell activation.

38. Kuhn, N. F. et al. CD40 ligand-modified chimeric antigen receptor $\mathrm{T}$ cells enhance antitumor function by eliciting an endogenous antitumor response. Cancer Cell 35, 473-488 (2019).

39. Anderson, M. E. \& Siahaan, T. J. Targeting ICAM-1/ LFA-1 interaction for controlling autoimmune diseases: designing peptide and small molecule inhibitors. Peptides 24, 487-501 (2003).

40. Hunter, C. A. $\&$ Jones, S. A. IL- 6 as a keystone cytokine in health and disease. Nat. Immunol. 16, 448-457 (2015)

41. Tanaka, T. et al. IL-6 in inflammation, immunity and disease. Cold Spring Harb. Perspect. Biol. 6, a016295 (2014).

42. Mishra, D. et al. Parabrachial interleukin-6 reduces body weight and food intake and increases thermogenesis to regulate energy metabolism. Cell Rep. 26, 3011-3026 (2019).

43. Dinarello, C. A. Immunological and inflammatory functions of the interleukin-1 family. Ann. Rev. Immunol. 27, 519-550 (2009).

44. Ricciotti, E. \& FitzGerald, G. A. Prostaglandins and inflammation. Arterioscler. Thromb. Vasc. Biol. 31 986-1000 (2011).

45. Garlanda, C., Dinarello, C. A. \& Mantovani, A. The interleukin-1 family: back to the future. Immunity 39 1003-1018 (2013)

46. Dinarello, C. A. Interleukin-1 in the pathogenesis and treatment of inflammatory diseases. Blood 117 3720-3732 (2011)

47. Liu, Y. et al. Gasdermin E-mediated target cell pyroptosis by CAR T cells triggers cytokine release syndrome. Sci. Immunol. 5, eaax7969 (2020).

48. Sachdeva, M., Duchateau, P., Depil, S., Poirot, L. \& Valton, J. Granulocyte-macrophage colony-stimulating factor inactivation in CAR T-cells prevents monocyte-dependent release of key cytokine release syndrome mediators. J. Biol. Chem. 294, 5430-5437 (2019).

49. Manz, M. G. Human-hemato-lymphoid-system mice: opportunities and challenges. Immunity 26, 537-54 (2007).

50. Sterner, R. M. et al. GM-CSF inhibition reduces cytokine release syndrome and neuroinflammation but enhances CAR-T cell function in xenografts. Blood 133, 697-709 (2019).
51. Pennica, D. et al. Human tumor necrosis factor: precursor structure, expression and homology to lymphotoxin. Nature 312, 724-729 (1984)

52. Li, J. et al. CD3 bispecific antibody-induced cytokine release is dispensable for cytotoxic $\mathrm{T}$ cell activity. Sci. Transl. Med. 11, eaax8861 (2019).

53. Lin, C. F. et al. IFN- $\gamma$ synergizes with LPS to induce nitric oxide biosynthesis through glycogen synthase kinase-3-inhibited IL-10. J. Cell. Biochem. 105 746-755 (2008).

54. Tötemeyer, S. et al. IFN- $\gamma$ enhances production of nitric oxide from macrophages via a mechanism that depends on nucleotide oligomerization domain-2. J. Immunol. 176, 4804-4810 (2006)

55. Capaldo, C. T. \& Nusrat, A. Cytokine regulation of tight junctions. Biochim. Biophys. Acta. 1788, 864-871 (2009)

56. Rahman, M. T. et al. IFN- $\gamma$, IL-17A, or zonulin rapidly increase the permeability of the blood-brain and small intestinal epithelial barriers: relevance for neuro-inflammatory diseases. Biochem. Biophys. Res. Commun. 507, 274-279 (2018)

57. Chai, Q., He, W. Q., Zhou, M., Lu, H. \& Fu, Z. F Enhancement of blood-brain barrier permeability and reduction of tight junction protein expression are modulated by chemokines/cytokines induced by rabies virus infection. J. Virol. 88, 4698-4710 (2014).

58. Lopez-Ramirez, M. A. et al. Role of caspases in cytokine-induced barrier breakdown in human brain endothelial cells. J. Immunol. 189, 3130-3139 (2012).

59. Volmar, A. M. The role of atrial natriuretic peptide in the immune system. Peptides 26, 1086-1094 (2005).

60. Staedtke, V. et al. Disruption of a self-amplifying catecholamine loop reduces cytokine release syndrome. Nature 564, 273-277 (2018).

61. Davila, M. L., Kloss, C. C., Gunset, G. \& Sadelain, M. CD19 CAR-targeted T cells induce long-term remission and $B$ cell aplasia in an immunocompetent mouse model of B cell acute lymphoblastic leukemia. PLOS ONE 8, e61338 (2013).

62. Bondanza, A. et al. Suicide gene therapy of graft versus-host disease induced by central memory human T lymphocytes. Blood 107, 1828-1836 (2006).

63. Mastaglio, S et al. NY-ESO-1 TCR single edited stem and central memory $T$ cells to treat multiple myeloma without graft-versus host disease. Blood 130, 606-618 (2017).

64. Siegler, E. L. \& Wang, P. Preclinical models in chimeric antigen receptor engineered T-cell therapy. Hum. Gene Ther. 29, 534-546 (2018).

65. Locke, F. L. et al. Preliminary results of prophylactic tocilizumab after axicabtageneciloleucel (axi-cel; KTE-C19) treatment for patients with refractory, aggressive non-hodgkin lymphoma (NHL). Blood 130 1547 (2017)

66. Dyson, A. \& Singer, M. Animal models of sepsis: why does preclinical efficacy fail to translate to the clinical setting? Crit. Care Med. 37, S30-S37 (2009).

67. Page, A. V. \& Liles, W. C. Biomarkers of endothelial activation/dysfunction in infectious diseases. Virulence 4, 507-516 (2013)

68. Schwameis, M., Schörgenhofer, C., Assinger, A. Steiner, M. M. \& Jilma, B. VWF excess and ADAMTS13 deficiency: a unifying pathomechanism linking inflammation to thrombosis in DIC, malaria, and TTP. Thromb. Haemost. 113, 708-718 (2015).

69. Gust, J. et al. Glial injury in neurotoxicity after pediatric CD19-directed chimeric antigen receptor T cell therapy. Ann. Neurol. 86, 42-54 (2019).

70. Kim, H. J., Tsao, J. W. \& Stanfill, A. G. The curren state of biomarkers of mild traumatic brain injury. JCl Insight 3, e97105 (2018).

71. Sofroniew, M. V. Multiple roles for astrocytes as effectors of cytokines and inflammatory mediators. Neuroscientist 20, 160-172 (2014).

72. Schuster, S. J. et al. Chimeric antigen receptor T cells in refractory B-cell lymphomas. N. Engl. J. Med. 377 , 2545-2554 (2017).

73. Gutierrez, E. G., Banks, W. A. \& Kastin, A. J. Blood-borne interleukin-1 receptor antagonist crosses the blood-brain barrier. J. Neuroimmunol. 55, 153-160 (1994).

74. Galea, J. et al. Intravenous anakinra can achieve experimentally effective concentrations in the central nervous system within a therapeutic time window: results of a dose-ranging study. J. Cereb. Blood Flow. Metab. 31, 439-447 (2011).
75. Parker, K. R. et al. Single-cell analyses identify brain mural cells expressing CD19 as potential off-tumor targets for CAR-T immunotherapies. Cell 183, 126-142.e17 (2020).

76. Dorovini-Zis, K. et al. The neuropathology of fatal cerebral malaria in Malawian children. Am. J. Pathol. 178, 2146-2158 (2011).

77. Olah, M. et al. Single cell RNA sequencing of human microglia uncovers a subset associated with Alzheimer's disease. Nat. Commun. 11, 6129 (2020).

78. Paolicelli, R. C. et al. Synaptic pruning by microglia is necessary for normal brain development. Science 333, 1456-1458 (2011)

79. Reu, P. et al. The lifespan and turnover of microglia in the human brain. Cell Rep. 20, 779-784 (2017).

80. Pluvinage, J. V. et al. CD22 blockade restores homeostatic microglial phagocytosis in ageing brains. Nature 568, 187-192 (2019)

81. DeAngelo, D. J. et al. Clinical outcomes for the phase 2, single-arm, multicenter trial of JCAR015 in adult B-ALL (ROCKET Study) [abstract P217]. SITC 2017 Annual Meeting Abstracts Book https:// higherlogicdownload.s3.amazonaws.com/SITCANCER 3bcb5ebf-803a-42fe-83b6-0773bc4eb962 Uploadedlmages/Annual\%20Meeting\%202017/SITC 2017 Abstract Book.pdf (2017).

82. Mahadeo, K. M. et al. Management guidelines for paediatric patients receiving chimeric antigen receptor T cell therapy. Nat. Rev. Clin. Oncol. 16 , 45-63 (2019)

83. Lee, D. W. et al. ASTCT consensus grading for cytokine release syndrome and neurologic toxicity associated with immune effector cells. Biol. Blood Marrow Transpl. 25, 625-638 (2019).

84. Maus, M. V. et al. Society for Immunotherapy of Cancer (SITC) clinical practice guideline on immune effector cell-related adverse events. J. Immunother. Cancer 8, e001511 (2020).

85. Nishimoto, N. et al. Mechanisms and pathologic significances in increase in serum interleukin-6 (IL-6) and soluble IL- 6 receptor after administration of an anti-IL-6 receptor antibody, tocilizumab, in patients with rheumatoid arthritis and Castleman disease. Blood 112, 3959-3964 (2008).

86. Nastoupil, L. J. et al. Standard-of-care axicabtagene ciloleucel for relapsed or refractory large B-cell lymphoma: results from the US lymphoma CAR T consortium. J. Clin. Oncol. 38, 3119-3128 (2020).

87. Strati, P. et al. Prognostic impact of dose, duration, and timing of corticosteroid therapy in patients with large B-cell lymphoma treated with standard of care axicabtagene ciloleucel (Axi-cel). J. Clin. Oncol. 38 8011 (2020).

88. Topp, M. V. et al. Earlier steroid use with axicabtagene ciloleucel (Axi-Cel) in patients with relapsed/refractory large B cell lymphoma. Blood 134, 243 (2019).

89. Siddiqi, T et al. Patient characteristics and pre-infusion biomarkers of inflammation correlate with clinical outcomes after treatment with the defined composition, CD 19-targeted CAR T cell product, JCAR017. Blood 130, 193 (2017).

90. Jacobson, C. A et al. Axicabtagene ciloleucel in the non-trial setting: outcomes and correlates of response, resistance, and toxicity. J. Clin. Oncol. 38 3095-3106 (2020).

91. Raje, N. et al. Anti-BCMA CAR T-cell therapy bb2121 in relapsed or refractory multiple myeloma. N. Engl. J. Med. 380, 1726-1737 (2019).

92. Porter, D. L. et al. Chimeric antigen receptor T cells persist and induce sustained remissions in relapsed refractory chronic lymphocytic leukemia. Sci. Transl. Med. 7, 303ra139 (2015).

93. Turtle, C. J. et al. Durable molecular remissions in chronic lymphocytic leukemia treated with CD19-specific chimeric antigen receptor-modified T cells after failure of ibrutinib. J. Clin. Oncol. 35 3010-3020 (2017).

94. Hirayama, A. V. et al. The response to lymphodepletion impacts PFS in patients with aggressive non-Hodgkin lymphoma treated with CD 19 CAR T cells. Blood 133, 1876-1887 (2019).

95. Kochenderfer, J. N. et al. Lymphoma remissions caused by anti-CD 19 chimeric antigen receptor T cells are associated with high serum interleukin- 15 levels. J. Clin. Oncol. 35, 1803-1813 (2017).

96. Neelapu, S. S. CAR-T efficacy: is conditioning the key? Blood 133, 1799-1800 (2019).

97. Neelapu, S. S. et al. Outcomes of older patients in ZUMA-1, a pivotal study of axicabtagene ciloleucel in refractory large B-cell lymphoma. Blood 135, 2106-2109 (2020). 
98. Ying, Z. et al. A safe and potent anti-CD19 CAR T cell therapy. Nat. Med. 25, 947-953 (2019). This study reports a novel CD19CAR construct with 4-1 BB and $C D 3 \zeta$ co-stimulatory domains that in clinical practice conferred protection against B cell lymphomas without increasing serum cytokine levels or inducing CRS

99. Ghorashian, S. et al. Enhanced CAR T cell expansion and prolonged persistence in pediatric patients with ALL treated with a low-affinity CD19 CAR. Nat. Med 25, 1408-1414 (2019).

This study reports a modified CD19CAR construct with lower binding affinity, resulting in both reduced CRS and increased expansion of CAR T cell populations in vivo.

100. Locke, F. L. et al. Long-term safety and activity of axicabtagene ciloleucel in refractory large B-cell lymphoma (ZUMA-1): a single-arm, multicenter phase 1-2 trial. Lancet Oncol. 20, 31-42 (2019).

101. Brudno, J. N. et al. Safety and feasibility of anti-CD19 CAR $T$ cells with fully human binding domains in patients with B-cell lymphoma. Nat. Med. 26 270-280 (2020).

102. Liu, E. et al. Use of CAR-transduced natural killer cells in CD19-positive lymphoid tumors. N. Engl. J. Med. 382, 545-553 (2020).

103. Feucht, J. et al. Calibration of CAR activation potential directs alternative $\mathrm{T}$ cell fates and therapeutic potency. Nat. Med. 25, 82-88 (2019). This study shows that altering the activation potential of a CAR construct can influence T cell differentiation and the balance between effector and memory $\mathrm{T}$ cell phenotypes.

104. Acharya, U. H. et al. Management of cytokine release syndrome and neurotoxicity in chimeric antigen receptor (CAR) T cell therapy. Exp. Rev. Hematol. 12 195-205 (2019)

105. Huarte, E. et al. Itacitinib (INCB039110), a JAK1 inhibitor, reduces cytokines associated with cytokine release syndrome induced by CAR T-cell therapy. Clin. Cancer Res. 26, 6299-6309 (2020).

106. Dubovsky, J. A. et al. Ibrutinib is an irreversible molecular inhibitor of ITK driving a $\mathrm{T}_{\mathrm{H}} 1$-selective pressure in T lymphocytes. Blood 122, 2539-2549 (2013).

107. Ruella, M. et al. Kinase inhibitor ibrutinib to prevent cytokine-release syndrome after anti-CD 19 chimeric antigen receptor $\mathrm{T}$ cells for B-cell neoplasms. Leukemia 31, 246-248 (2017)

108. Mestermann, K. et al. The tyrosine kinase inhibitor dasatinib acts as a pharmacologic on/off switch for CAR T cells. Sci. Transl. Med. 11, eaau5907 (2019).

109. Sun, C. et al. THEMIS-SHP1 recruitment by 4-1BB tunes LCK-mediated priming of chimeric antigen receptor-redirected T cells. Cancer Cell 37, 216-225. e6 (2020)

110. Strati, P. et al. Clinical efficacy of anakinra to mitigate CAR T-cell therapy-associated toxicity in large B-cell lymphoma. Blood Adv. 4, 3123-3127 (2020).

111. Henter, J. I. et al. Emapalumab in primary hemophagocytic lymphohistiocytosis. N. Engl. J. Med. 383, 596-598 (2020).

112. Boardman, D. A. et al. Expression of a chimeric antigen receptor specific for donor HLA class I enhances the potency of human regulatory T cells in preventing human skin transplant rejection. Am. J. Transplant. 17, 931-943 (2017).

113. Boroughs, A. C. et al. Chimeric antigen receptor costimulation domains modulate human regulatory T cell function. JCI Insight 5, e 126194 (2019).

114. Dawson, N. A. J. et al. Functional effects of chimeric antigen receptor co-receptor signaling domains in human regulatory T cells. Sci. Transl. Med. 12, eaaz3866 (2020)

115. Rosado-Sánchez, I. \& Levings, M. K. Building a CAR-T $\mathrm{T}_{\text {reg }}$ : going from the basic to the luxury model. Cell. Immunol. 358, 104220 (2020).
116. Jatiani, S. S. et al. Myeloma CAR-T CRS management with IL-1R antagonist anakinra. Clin. Lymphoma Myeloma Leuk. 20, 632-636.e1 (2020).

117. Voskoboinik, I. et al. Perforin and granzymes: function dysfunction and human pathology. Nat. Rev. Immunol. 15, 388-400 (2015).

118. Grom, A. A., Horne, A. \& De Benedetti, F. Macrophage activation syndrome in the era of biologic therapy. Nat. Rev. Rheumatol. 12, 259-268 (2016).

119. Gadoury-Levesque, V. et al. Frequency and spectrum of disease-causing variants in 1892 patients with suspected genetic HLH disorders. Blood Adv. 4 2578-2594 (2020).

120. Teachey, D. T. et al. Toxicity management after chimeric antigen receptor $\mathrm{T}$ cell therapy: one size does not fit 'ALL'. Nat. Rev. Clin. Oncol. 15, 218 (2018)

121. Teachey, D. T. et al. Cytokine release syndrome after blinatumomab treatment related to abnormal macrophage activation and ameliorated with cytokinedirected therapy. Blood 121, 5154-5157 (2013).

122. Ishii, K. et al. Perforin-deficient CAR T cells recapitulate late-onset inflammatory toxicities observed in patients. J. Clin. Invest. 130, 5425-5443 (2020).

123. Sumegi, J. et al. Gene expression profiling of peripheral blood mononuclear cells from children with active hemophagocytic lymphohistiocytosis. Blood 117, e151-e160 (2011).

124. Canna, S. W. et al. An activating NLRC4 inflammasome mutation causes autoinflammation with recurrent macrophage activation syndrome. Nat. Genet. 46 1140-1146 (2014).

125. Moore, J. B. \& June, C. H. Cytokine release syndrome in severe COVID-19. Science 368, 473-474 (2020).

126. Ruan, Q., Yang, K., Wang, W., Jiang, L. \& Song, J. Clinical predictors of mortality due to COVID-19 based on an analysis of data of 150 patients from Wuhan, China. Intensive Care Med. 46, 846-848 (2020).

127. Chen, G. et al. Clinical and immunological features of severe and moderate coronavirus disease 2019. J. Clin. Invest. 130, 2620-2629 (2020).

128. Guaraldi, G. et al. Tocilizumab in patients with severe COVID-19: a retrospective cohort study. Lancet Rheumatol. 2, e474-e484 (2020).

129. Xu, X. et al. Effective treatment of severe COVID-19 patients with tocilizumab. Proc. Natl Acad. Sci. USA 117, 10970-10975 (2020).

130. Campochiaro, C. et al. Efficacy and safety of tocilizumab in severe COVID-19 patients: single-center retrospective cohort study. Eur. J. Intern. Med. 76, 43-49 (2020)

131. Salama, C. et al. Tocilizumab in patients hospitalized with COVID-19 pneumonia. N. Engl. J. Med. 384 , 20-30 (2021)

132. Kewan, T. et al. Tocilizumab for treatment of patients with severe COVID-19: a retrospective cohort study. EClinicalMedicine 24, 100418 (2020).

133. Huet, T. et al. Anakinra for severe forms of COVID-19: a cohort study. Lancet Rheumatol. 2, e393-e400 (2020).

134. Cavalli, G. et al. Interleukin-1 blockade with high-dose anakinra in patients with COVID-19, acute respiratory distress syndrome, and hyperinflammation: retrospective cohort study. Lancet Rheumatol. 2 e325-e331 (2020)

135. Bozzi, G. et al. Anakinra combined with methylprednisolone in patients with severe COVID-19 pneumonia and hyperinflammation: an observational cohort study. J. Allergy Clin. Immunol. 147, 561-566.e4 (2021)

136. Cauchois, R. et al. Early IL-1 receptor blockade in severe inflammatory respiratory failure complicating COVID-19. Proc. Natl Acad. Sci. USA 117 18951-18953 (2020).

137. Amor, C. et al. Senolytic CAR T cells reverse senescence-associated pathologies. Nature $\mathbf{5 8 3}$, 127-132 (2020)
138. Dinarello, C. A. Why not treat human cancer with interleukin-1 blockade? Cancer Metastasis Rev. 29 317-329 (2010)

139. Voronov, E. et al. IL-1 is required for tumor nvasiveness and angiogenesis. Proc. Natl Acad. Sci. USA 100, 2645-2650 (2003).

140. Horikawa, N. et al. Expression of vascular endothelial growth factor in ovarian cancer inhibits tumor immunity through the accumulation of myeloid-derived suppressor cells. Clin. Cancer Res. 23, 587-599 (2017).

141. Voronov, E., Yaron, C. \& Apte, R. N. The role IL-1 in tumor-mediated angiogenesis. Front. Physiol. 5, 114 (2014).

142. Kaplanov, I. et al. Blocking IL-1 $\beta$ reverses the immunosuppression in mouse breast cancer and synergizes with anti-PD-1 for tumor abrogation. Proc. Natl Acad. Sci. USA 116, 1361-1369 (2019).

143. Carey, A. et al. Identification of interleukin- 1 by functional screening as a key mediator of cellular expansion and disease progression in acute myeloid eukemia. Cell Rep 18, 3204-3218 (2017).

144. Ridker, P. M. et al. Anti-inflammatory therapy with canakinumab for atherosclerotic disease. N. Engl. J. Med. 377, 1119-1131 (2017).

145. Ridker, P. M. et al. Effect of interleukin-1 $\beta$ inhibition with canakinumab on incident lung cancer in patients with atherosclerosis: exploratory results from a randomised, double-blind, placebo-controlled trial. Lancet 390, 1833-1842 (2017).

146. Litmanovich, A., Khazim, K. \& Cohen, I. The role of interleukin-1 in the pathogenesis of cancer and its potential as a therapeutic target in clinical practice. Oncol. Ther. 6, 109-127 (2018)

Acknowledgements

E.C.M. was supported by the National Institute for Health Research (NIHR) University College London Hospitals (UCLH) Biomedical Research Centre.

Author contributions

The authors contributed equally to all aspects of the article.

\section{Competing interests}

M.S. and T.G. are listed as inventors in a Memorial Sloan Kettering-held patent application related to treatment of cytokine release syndrome: 'Methods and compositions for alleviating cytokine release syndrome' (WO2019099993A1). E.C.M. is scientific co-founder of Quell Therapeutics Ltd, which is developing chimeric antigen receptor (CAR)-modified regulatory $\mathrm{T}$ cells, and has served as a consultant to Kite, a Gilead company. S.S.N. served as consultant to Kite, a Gilead Company, Merck, Bristol-Myers Squibb, Novartis, Celgene, Pfizer, Allogene Therapeutics, Cell Medica/Kuur, Incyte, Precision Biosciences, Legend Biotech, Adicet Bio, Calibr and Unum Therapeutics; received research support from Kite, a Gilead Company, Bristol-Myers Squibb, Merck, Poseida, Cellectis, Celgene, Karus Therapeutics, Unum Therapeutics, Allogene Therapeutics, Precision Biosciences and Acerta; received royalties from Takeda Pharmaceuticals; and has intellectual property related to cell therapy.

\section{Peer review information}

Nature Reviews Immunology thanks A. Bondanza, S. Grupp and C. Jacobson for their contribution to the peer review of this work.

Publisher's note

Springer Nature remains neutral with regard to jurisdictional claims in published maps and institutional affiliations.

RELATED LINKS

ClinicalTrials.gov: https://clinicaltrials.gov/

(C) Springer Nature Limited 2021 